\title{
LOCAL DEGREE OF SEPARABILITY AND INVARIANCE OF DOMAIN
}

\author{
L. B. TREYBIG
}

\begin{abstract}
In $E^{n}$ an invariance of domain theorem may be proved assuming the Jordan Brouwer Theorem. In this paper such a theorem is proved for various locally compact, connected, Hausdorff spaces which satisfy a certain local degree of separability property. An example shows the separability condition may not be removed. A second theorem yields additional information about homogeneous spaces which satisfy the hypotheses of the first theorem.
\end{abstract}

In ([2], [3], [4]) the invariance of domain for $n$-manifolds is proved using either essential mappings or the Jordan Brouwer Theorem. Thelatter proof is generalized in Theorem 1 to certain locally compact, connected Hausdorff spaces by adding hypotheses concerning local degree of separability. Curiously enough, such a condition is necessary in the sense that there is a counterexample (Example 1) to Theorem 1 if the separability condition is omitted. Theorem 2 shows that if a homogeneous space $X$ satisfies the conditions of Theorem 1 plus two other restrictions, then $X$ is first countable and locally separable.

The space $X$ will be said to have the invariance of domain property if given $h: U \rightarrow X$ a homeomorphism of an open subset $U$ of $X$ into $X$, then $h(U)$ is open. The local degree of separability, l.s. $(p)$, of $X$ at $p \in X$ is the least cardinal $k$ such that an open neighborhood of $p$ contains a dense subset $B$ with card $B \leqq k$.

THEOREM 1. Let $(X, T)$ be a locally compact, connected Hausdorff space such that if $a \in U \in T$ and $b \in X-U$, then there is a collection $C$ of mutually exclusive continua such that (1) $a \in \bigcup C \subset U$, where $\bigcup C$ is connected and open, (2) if $a \in g_{0} \in C$ and $g \in C-\left\{g_{0}\right\}$, then $g$ separates $a$ from $b$ in $X$, (3) if $h: \cup C \rightarrow X$ is a homeomorphism into and $g \in C-\left\{g_{0}\right\}$ then $g$ contains a subcontinuum $g^{\prime}$ such that $X-h\left(g^{\prime}\right)$ is not connected, and (4) card $C>1 . s .(p)$ for each $p \in X$. Then $X$ has the invariance of domain property.

Received by the editors March 5, 1970.

AMS 1969 subject classifications. Primary 5460; Secondary 5440, 5478.

Key words and phrases. Invariance of domain, local degree of separability, homogeneous space.

(c) American Mathematical Society 1972 
Proof. Suppose $U \in T$ and $h: U \rightarrow X$ is a homeomorphism into, but that $y \in h(U) \cap \mathrm{Cl}(X-h(U))$. We may also suppose without loss of generality that $U$ is connected. Let $x=h^{-1}(y)$ and $W \in T$ such that $y \in W \subset \bar{W} \subset X-z$, where $z \in h(U)$, and $\bar{W}$ and $\bar{W} \cap h(U)$ are both compact.

By the hypothesis there is a connected open set $W_{1}$ so that $y \in W_{1} \subset$ $\bar{W}_{1} \subset W$. Some subcontinuum $A$ of $\bar{W}_{1}$ is irreducible between a point $t$ of $\bar{W}_{1}-h(U)$ and $\bar{W}_{1} \cap h(U) . A-h(U)$ is connected and has a point $s$ of $h(U)$ in its closure. Letting $s=a, b=t, U-t$, we find a collection $C^{\prime}$ of continua as guaranteed in the hypothesis. But since $\bigcup C^{\prime}$ is open, some element $B$ of $C^{\prime}$ separates $s$ from $t$ in $X$ and also intersects $A-h(U)$ and $h(U) \cap \bar{W}$. Some subcontinuum $B^{\prime}$ of $B$ is irreducible between a point of $B \cap(A-h(U))$ and $B \cap h(U) . B^{\prime}-h(U)$ has a point $r$ of $h(U)$ in its closure, where $r \neq s$. Thus $D=\left(A \cup B^{\prime}\right)-h(U)$ is a connected subset of $X-h(U)$ with points $r, s$ of $h(U) \cap W$ in its closure.

Let $s \in M \in T$ where $M$ has a dense subset $N$ where card $N=1$.s. $(s)$. Let $a, b, V=\bigcup C$, and $C$ be as in the hypothesis where $a=h^{-1}(s), b=$ $h^{-1}(r)$, and $V \subset U \cap\left(h^{-1}\left(M \cap W_{1}-r\right)\right)$. Assume $a \in g_{0} \in C$, and for each $g \in C-\left\{g_{0}\right\}$ let $g^{\prime}$ denote a subcontinuum of $g$ such that $X-h\left(g^{\prime}\right)$ is the union of two mutually separated sets $R_{g^{\prime}}$ and $S_{g^{\prime}}$, where $s \notin R_{g^{\prime}}$.

Now suppose $g_{1}$ and $g_{2}$ are two elements of $C$ such that $g_{1}$ separates $g_{2}$ from $a$ in $V$. (Note that the methods of Theorem 81, p. 33 of [5] reveal that $C-\left\{g_{0}\right\}$ is totally ordered under the relation $g<g^{\prime}$ if and only if $g$ separates $a$ from $g^{\prime}$ in $X$; in fact, with the topology induced by $<C-\left\{g_{0}\right\}$ it is also connected.) If $R_{g_{1}}$ and $R_{g_{2}}{ }^{\prime}$ intersect, then $h\left(g_{1}^{\prime}\right) \notin R_{g_{2}}$; for otherwise it would then follow that $g_{2}^{\prime}$ separates $g_{1}^{\prime}$ from $a$ in $U$; and thus $g_{2}$ would separate $g_{1}$ from $a$ in $X$, a contradiction. Therefore $h\left(g_{2}^{\prime}\right) \cup R_{g_{2}}, \subset R_{g_{1}}$. Let $U_{1}$ denote the complementary domain of $U-g_{2}$ containing $h^{-1}(r)$. But $D \cup\{r, s\} \cup h\left(U_{1} \cup g_{2}\right)$ is a connected subset of $X-h\left(g_{1}^{\prime}\right)$ which contains $s$ and a point of $R_{g_{1}{ }^{\prime}}$, a contradiction. Thus $R_{g_{1^{\prime}}} \subset X-R_{g_{2}}$.

Finally, $L=\left\{M \cap R_{g^{\prime}}: g \in C\right\}$ is a collection of disjoint open subsets of $M$ where card $L=$ card $C>$ l.s. $(s)$. Since each element of $L$ contains an element of $N$ it follows that card $N \geqq$ card $C$, so l.s. $(s) \geqq$ card $C$, a contradiction.

Corollary 1. A locally compact Moore space satisfying Axioms 0-5 of [5] has the invariance of domain property.

Proof. This follows from Theorem 1 with the aid of Theorem 58, p. 23 and Theorem 14, p. 171 of [5].

REMARK. In Theorem 1 if $X$ is a $n$-manifold, then for each $x \in U$ open let $k$ be a homeomorphism from $V \subset U$ onto the open unit ball in $R^{n}$, where $k(x)=0$. Let $C=\{\{x\}\} \cup\left\{k^{-1}(S): S\right.$ is a sphere in $R^{n}$ with center 0 and radius less than 1$\}$. 
Lemma 1. Suppose $a, b, U, C, V=\bigcup C$ are as in the hypothesis of Theorem 1 , and $a \in g_{0} \in C$ and $C-\left\{g_{0}\right\}$ is totally ordered under the relation $\leqq$ described above. Then, if for each $g \in C-\left\{g_{0}\right\}$, the set $X-g=R_{g} \cup S_{g}$ mutually separated where $R_{g}$ is the component of $X-g$ containing a then (1) there is a $g \in C-\left\{g_{0}\right\}$ so that if $g^{\prime} \leqq g$ then $g^{\prime} \cup R_{g^{\prime}} \subset V$ and (2) if $W$ is an open set containing $g_{0}$ then there exists $g \in C-\left\{g_{0}\right\}$ such that $R_{g} \cup g \subset W$.

Proof. Since $X$ is locally connected, there is no harm in assuming each $R_{g}$ above is the component of $X-g$ containing $a$. Note from above that $g<g^{\prime}$ implies $g \cup R_{g} \subset R_{g^{\prime}}$. Let $M=\bigcap_{g_{\in} C-\left\{g_{0}\right\}} R_{g}=\bigcap_{g_{\in} C-\left\{g_{0}\right\}} g \cup R_{g}=$ $\bar{M}$. Suppose $M-g_{0}$ is not void. Since $M-g_{0} \subset X-V$ and $g_{0}$ is closed, then $g_{0}$ and $M-g_{0}$ are mutually separated. Since $X$ is connected let $x \in$ $\left(M-g_{0}\right) \cap \mathrm{Cl}\left(\cup S_{g}\right)$. Let $W_{0}$ be a connected open set containing $x$ so that $\bar{W}_{0}$ is a compact subset of $X-g_{0}$ and let $g \in C-\left\{g_{0}\right\}$ such that $W_{0}$ intersects $S_{g}$. But $W_{0}$ must intersect $g$ since otherwise $W_{0} \subset S_{g}$. Thus $W_{0}$ intersects $S_{g^{\prime}}$ for all $g^{\prime} \leqq g$. Using connected open subsets of $X-g_{0}$ whose closures are compact, and which intersect $g \cup S_{g}$, a chain argument yields a continuum $N$ so that $b, x \in N \subset X-g_{0}$. Thus if $g^{\prime} \leqq g$ then $g^{\prime}$ intersects $N$.

There is an open set $R$ containing $g_{0}$ so that $\bar{R}$ is a compact subset of $X-\left(N \cup M-g_{0}\right)$. For every $g_{1} \leqq g$ there is a $g^{\prime} \leqq g_{1}$ so that $g^{\prime}$ intersects $R$ and also $N$. Thus, there is a point $t$ of $\mathrm{Bd} R$ so that if $t \in Q \in T$ and $g_{1} \leqq g$ then there exists $g^{\prime} \leqq g_{1}$ such that $g^{\prime}$ intersects $Q$. Since $t \notin M$, $t \in S_{g^{\prime}}$, for some $g^{\prime}$. But if $g^{\prime \prime}<g^{\prime}$ then $g^{\prime \prime}$ does not intersect $Q=S_{g^{\prime}}$, a contradiction. Thus $g_{0}=M$.

In part (2) suppose $W_{1}$ is an open set such that $g_{0} \subset W_{1} \subset W$, where $\bar{W}_{1}$ is compact. There is a finite set $\left\{g_{1}, \cdots, g_{n}\right\}$ of elements of $C-\left\{g_{0}\right\}$ such that $\bigcup_{p=1}^{n} S_{g_{p}}$ covers $\mathrm{Bd}\left(W_{1}\right)$. Let $g_{i}^{\prime}$ denote the least of these in the order $\leqq$. Since $g_{i} \cup R_{g_{i}}$ is connected and contains $a$ but no point of $\operatorname{Bd}\left(W_{1}\right)$, then $g_{i} \cup R_{g_{i}} \subset W_{1} \subset W$.

THEOREM 2. If (1) $(X, T)$ is as in Theorem 1 and is homogeneous, (2) $\aleph_{1}=2^{\boldsymbol{N}_{0}}$ and (3) for each such $a, b, U$ described in Theorem 1 the element $g_{0}$ of $C$ which contains $a$ is $\{a\}$, then $X$ is locally separable and first countable.

Proof. Let $a, b, U, C, V=\bigcup C$ be as in Theorem 1 where $\bar{V}$ is compact and $a \in g_{0} \in C$. Let $g, g_{1}, g_{2}, \cdots$ be a sequence of elements of $C-\left\{g_{0}\right\}$ such that $g<g_{p+1}<g_{p}, p=1,2, \cdots$. There exists $g^{\prime} \in C-\left\{g_{0}\right\}$ so that $g^{\prime}$ is the g.l.b. $\left\{g_{1}, g_{2}, \cdots\right\}$ and a point $x$ of $g^{\prime}$ so that every open set containing $x$ intersects infinitely many $g_{i}$ 's.

Let $C^{\prime}$ be as in Theorem 1 for $a^{\prime}=x, b^{\prime}=b, U^{\prime}=U$, and let $V^{\prime}=\bigcup C^{\prime}$ and $x \in h_{0} \in C^{\prime}$. For each $n$ let $U_{n}=X-g_{n}$ and for each $h \in C^{\prime}-\left\{h_{0}\right\}$ let $X-h=R_{h} \cup S_{h}$ mutually separated, where $x \in R_{h}$ and $R_{h}$ is connected. Let $C^{\prime}-\left\{h_{0}\right\}$ be ordered as above. Let elements $h_{1}, h_{2}, \cdots$ of $C^{\prime}-\left\{h_{0}\right\}$ be chosen such that $h_{n} \cup R_{h_{n}} \subset U_{n}$ and $h_{n+1}<h_{n}$ for $n=1,2, \cdots$. 
Suppose $x \in Q \in T$. But by Lemma 1 (since $\{x\}=h_{0}$ ) there is an $n$ so that $R_{h_{n}} \cup h_{n} \subset Q$. Thus, $X$ has a countable base at $x$, so by homogeneity has one at each point.

Let $y \in g^{\prime}$ such that every open set containing $y$ intersects a $g^{\prime \prime}$ for $g^{\prime \prime}<g^{\prime}$, and let $R_{1}, R_{2}, \cdots$ denote a countable base at $y$. Select elements $k_{1}, k_{2}, \cdots$ of $C-\left\{g_{0}\right\}$ such that $k_{n}$ intersects $R_{n}$ and $k_{n}<k_{n+1}$. The open segments $\left(k_{n}, g_{n}\right)$ form a countable base at $g^{\prime}$ in the connected totally ordered set $C-\left\{g_{0}\right\}$. Analogous double use of the countable base at a point in $X$ will produce for any $g^{\prime \prime} \in\left(C-\left\{g_{0}\right\}\right.$, $\left.\leqq\right)$ a countable base. By a theorem of Babcock [1] $\operatorname{card}\left(C-\left\{g_{0}\right\}\right) \leqq 2^{N_{0}}$. By the continuum hypothesis $1 . s .(p) \leqq$ $\aleph_{0}$ for each $p \in X$.

BACKGROUND. Given a well-ordered sequence $\alpha$ and a totally ordered set $B$ let $B^{\alpha}$ denote the set of all sequences isomorphic to $\alpha$, each term of which is in $B$, and let $B^{\alpha}$ be understood to have the lexicographic order. Let $L_{0}=L=[0,1]$ and let $\alpha_{1}=1,2,3, \cdots$. Also, let $\alpha_{2}=\alpha_{1}{ }^{\alpha_{1}}$ and let $L_{i}=L^{\alpha_{i}}(i=1,2)$.

It is known (Babcock [1]) that if $J$ denotes one of $L_{0}, L_{1}$, and $L_{2}$, then in the interval topology (1) $J$ is compact, connected, and first countable, and (2) every pair of subintervals of $J$ are homeomorphic. Furthermore, no two of $L_{0}, L_{1}$ and $L_{2}$ are homeomorphic. Let $L_{p}=a_{p} b_{p}, p=0,1,2$.

LEMMA 2. Let $G$ denote an upper semicontinuous decomposition of $L_{2} \times L_{2}$ such that $g \in G$ provided $(1) g=\{(a, b)\}$ where $a, b \in L_{2}$ and $b>a_{2}$, or (2) there is an element $\left(a, a_{2}\right)$ of $L_{2} \times L_{2}$ such that $g=\left\{\left(a^{\prime}, a_{2}\right)\right.$ in $L_{2} \times L_{2}$ so that $a$ and $a^{\prime}$ agree on all coordinates not preceded by an infinite number of coordinates $\}$. Then, there is no homeomorphism of $\left(L_{2} \times L_{2}\right) / G$ into $L_{2} \times L_{2}$.

Proof. Suppose there is such a homeomorphism $h$. Let $c_{1}, c_{2}, \cdots$ denote a sequence of elements of $L_{2}$ which converge to $a_{2}$, and where $c_{p+1}<c_{p}$ for $p=1,2, \cdots$. Let $d_{p}=h\left(L_{2} \times\left\{c_{p}\right\}\right), p=1,2, \cdots$ and let $d_{0}=$ image of the nondegenerate elements of $G$ under $h$. Since $d_{0}$ is homeomorphic to $L_{1}, d_{0}$ contains no interval of the form $\{a\} \times K$ or $H \times\{b\}$, so let $e$ denote a "subarc" of $d_{0}$ containing no points with a coordinate $=a_{2}$ or $b_{2}$.

For each $n$ let $G_{n}$ denote a finite cover of $e$ by sets of the form $P=H \times K$, where each of $H$ and $K$ is an open subinterval of $L_{2}$, and where $P \subset L_{2} \times$ $L_{2}-d_{n}$. Let $C_{n}$ denote the set of all components $C$ of sets of the type $e \cap P, P \in G_{n}$, and let $K_{C}$ denote a set composed of the endpoints of $C$ and one point interior to $C$. For each $n$, let $H_{n}=\bigcup K_{C}, C \in C_{n}$.

In order to show each $C_{n}$ is countable it is helpful to use (1) the fact that $L_{2} \times L_{2}$ is first countable and (2) the fact that no generalized arc $A$ has the property that there are mutually exclusive closed sets $M, N$ and an infinite set $T$ of mutually exclusive segments of $A$ such that each $t \in T$ has one endpoint in $M$ and the other in $N$. Finally, to show $\bigcup H_{n}$ is dense in $e$ it 
must be remembered that $e$ contains no "vertical" or "horizontal" intervals. Since $\bigcup H_{n}$ is a countable set dense in $e$, this means $L_{\mathbf{1}}$ is homeomorphic to $L_{0}$, a contradiction.

EXAMPLE 1. There is a space $(X, T)$ satisfying all but condition (4) of the hypothesis of Theorem 1, and such that $X$ does not have the invariance of domain property.

Proof. Before we describe the example we need to describe some further decompositions of $L_{2} \times L_{2}$. Let $G$ be as in Lemma 2. Let $H$ be a decomposition of $L_{2} \times L_{2}$ so that $H$ agrees with $G$ on points $(a, b)$ with $b<b_{2}$, but on $L_{2} \times\left\{b_{2}\right\}$ let $\left(a, b_{2}\right)$ and $\left(a^{\prime}, b_{2}\right)$ belong to the same element of $H$ if and only if $a$ and $a^{\prime}$ have the same first coordinate. Let $K$ be defined so that $g \in K$ if and only if (1) $g$ is an element of $H$ containing no point of the form $\left(a_{2}, x\right)$ or $\left(b_{2}, x\right)$, or (2) there is an $x$ in $L_{2}$ so that $g$ is the union of the elements of $H$ containing $\left(a_{2}, x\right)$ and $\left(b_{2}, x\right)$, respectively. The set $A=$ $\left(L_{2} \times L_{2}\right) / K$ is a "generalized annulus" with a metric simple closed curve on one edge $E_{J}^{0}$ and a "simple closed curve" on the other edge $E_{J}^{1}$, which is the union of two $I_{1}$ arcs. Given a subset $M$ of $A$ let $P_{1}(M)$ denote the set of all elements $k$ of $K$ so that there is an element $m$ of $M$, where $k$ contains an element of the form $\left(a, a_{2}\right)$ and $m$ contains an element of the form $(a, x)$. Likewise, define $P_{0}(M)$ for points on the other edge. Note that if $m$ is a subset of the metric edge, and $N$ is the set of all points $\left(x, a_{2}\right)$ so that $\left(x, b_{2}\right) \in m \in M$, then $N$ is the union of elements of $K$.

The space $X$ will denote the Euclidean plane $R^{2}$ together with the union of a set of "annuli" $A_{J}$, one for each simple closed curve $J$ in the plane. The metric edge of $A_{J}$ is identified with $J$ under an identification map $i_{J}$ : $E_{J}^{0} \rightarrow J$, and if $J \neq J^{\prime}$, then $A_{J} \cap A_{J^{\prime}}=J \cap J^{\prime}$.

The topology $T$ for $X$ is generated by neighborhoods of the following type: If $x \in A_{J}-J$, let small open neighborhoods of $x$ be those in the decomposition space topology on $A_{J}$. If $x \in R^{2}$, a neighborhood $U$ of $x$ will be determined by (1) an $\varepsilon>0$, (2) the collection $V$ of all simple closed curves $J$ which intersect the spherical open set $N(x, \varepsilon)$, and (3) a collection $W$ of connected open subsets $S_{J}$, one for each $L_{2 J}$ (Jth copy of $L_{2}$ ) such that $J \in V$ and such that (1) $S_{J}$ contains the $b_{2 J}$ endpoint and (2) $S_{J}=$ $L_{2 J}$ for all but finitely many $J$ 's in $W$. $U$ is $\{p:(1) p \in N(x, \varepsilon)$ or (2) there is a $J \in V$, a point $q \in J \cap N(x, \varepsilon)$, and a point $(r, s)$ of $I_{2 J} \times I_{2 J}$ such that $(r, s) \in p, s \in S_{J}$, and $\left.i_{J}\left(P_{0}(p)\right)=q\right\}$.

We now see how to define the various collections $C$ of continua. Let $a \in U$ open and $b \in X-U$.

Case 1. Suppose $a \in E_{J}^{1}$. We think of $L_{2}$ as the $J$ th copy and of $K$ as the corresponding decomposition of $L_{2} \times L_{2}$. Let $x_{1}, x_{2}$ be two elements of $E_{J}^{1}$ distinct from $a$, suppose $a_{2}<W<b_{2}$ and suppose $B$ is the "arc" from $x_{1}$ to $x_{2}$ on $E_{J}^{1}$ that contains $a$. Let $g$ be the $\left\{P:(1) P=\{(x, w)\}\right.$ and $P_{1}(P) \in B$, 
or (2) $P=\{(x, y)\}$ and $a_{2}<y \leqq W$ and $P_{1}(P)=x_{1}$ or $x_{2}$, or (3) $P=x_{1}$ or $\left.x_{2}\right\}$. Continua such as $g$ (type $g$ ) will be used to construct $C$, although not all continua in $C$ will be of this type.

Let $U_{1}, U_{2}, \cdots$ denote a countable base of neighborhoods at $a$, where $U_{1} \subset U$. Let $g_{0}$ be a continuum of type $g$ so that $g \cup$ (the component of $A_{J}-g$ that contains $\left.a\right) \subset U_{1}$. Let $g_{1}=\{a\}$ and let $g_{1 / 2}$ be a type $g$ continuum so that $g_{1 / 2} \subset U_{2}$ and also separates $g_{0}$ from $a$ in $A_{J}$. Analogously, we find $g_{1 / 4}$ and $g_{3 / 4}$ so that $g_{3 / 4} \subset U_{3}$ and separates $g_{1 / 2}$ from $a$ and where $g_{1 / 4}$ separates $g_{0}$ from $g_{1 / 2}$. This process is continued to find for each $r=p / 2^{q}$ $(0 \leqq r<1)$ a continuum of type $g$, where the separations occur in the same way as on the real line, and where $g_{r} \subset U_{a+1}$ for $r=2^{q}-1 / 2^{q}$. If $0<t<1$ and $t \neq p / 2^{a}$ then $g_{t}$ is the set of all points of $A_{J}$ that are separated from $g_{0}$ by a previously defined $g_{s}$, for $s<t$, but are not separated from $g_{0}$ by such a $g_{s}$ for $s>t$. $C=\left\{g_{t}: 1 \geqq t>0\right\}$.

Case 2. If $a \in A_{J}-\left(E_{J}^{0} \cup E_{J}^{1}\right)$, then a proof analogous to that in Case 1 may be used. The continua will have four "sides" instead of three.

Case 3. Suppose $a \in R^{2}$ and let $U_{1} \subset U$ be determined by $\varepsilon, V$, and $W$ as in the definition of this type of neighborhood above. Let $s_{J_{1}}, \cdots, s_{J_{n}}$ be the sets in $W$ which are different from the corresponding $L_{J}$. For each $J_{p}$ $(p=1, \cdots, n)$ let $h_{p}$ denote a set valued map so that if $t \in[0, \varepsilon]$ then $h_{p}(t)$ is the set of all $w$ in $I_{2 J_{p}}$ whose first coordinate is $(1 / \varepsilon)\left(\varepsilon c_{p}+\left(d_{p}-c_{p}\right) t\right)$ $\left(c_{p}<d_{p}\right)$ and where every point in $L_{2 J_{p}}$ with first coordinate in $\left[c_{p}, d_{p}\right]$ is in $s_{J_{p}}$.

For $1>t>0$ let $g_{t}=\left\{P\right.$ : (i) $P \in R^{2}$ and $|P-a|=\varepsilon t$, or (ii) $P \in A_{J}, J \in W$, $s_{J}=I_{2 J}$, and $\left|P_{J}^{0}(P)-a\right|=\varepsilon t$, or (iii) $P \in J_{m}(1 \leqq m \leqq n)$ and $(\mathrm{a})\left|P_{J_{m}}^{0}(P)-a\right|=$ $\varepsilon t$ and $P=\{(x, y)\}$, where $y \geqq V \in h_{m}(t \varepsilon)$, or (b) there is a component $C$ of $J_{m}-\left\{Q \in R^{2}:|Q-a|=\varepsilon t\right\}$ such that $P=\{(x, y)\}$ and $P_{J_{m}}^{0}(P) \in C$ and $\left.y \in h_{m}(t \varepsilon)\right\}$. The set $g_{0}$ is defined to be the closure of the component of $X-$ $\cup g_{t}(0<t<1)$ that contains $a$.

To verify that condition (3) of the hypothesis holds, note that an application of Lemma 2 reveals that if $h: V \rightarrow X$ is a homeomorphism into, where $U$ is an open subset of a $A_{J}-J$ containing a segment $s$ of $E_{J}^{1}$, then $h(s)$ is a segment of some $E_{J}^{1}$. That $h\left(g_{t}\right)$ (Case 1 ) separates $X$ is a consequence of the work of Slye [6] applied to two sets of the form $A_{J}-E_{J}^{0}$ joined along a common edge $E_{J}^{1}$. In Case 2 the work of Slye may be applied to $A_{J}-\left(E_{J}^{0} \cup E_{J}^{1}\right)$. In Case 3 each $g_{t}(0<t<1)$ contains a simple closed curve $J \subset R^{2}$, and $h(J)$ separates $X$ into $A_{J}-J$ and $X-A_{J}$.

Let $W=(-1,1) \times(-1,1) \subset R^{2}$ be an open square disk and let $Q$ denote the set of all closed curves in $R^{2}$ which intersect $W$. Let $U=\{P \in X:$ (1) $P \in W$ or (2) $P \in A_{J}, J \in Q$ and $\left.P_{J}^{0}(P) \in W\right\}$. For each positive integer $n$ let $J_{n}$ be the rectangular simple closed curve with vertices at $(-n, 0),(n, 0)$, $(n, n)$ and $(-n, n)$, respectively, and let $C_{n}$ denote the set of all points $P$ 
of $A_{J_{n}}$ so that $P_{J_{n}}^{0}(P) \in W$. We define an into homeomorphism $h: U \rightarrow U$ which is the identity on $U-\cup C_{n}$ and such that $h\left(C_{n}\right)=C_{n+1}$. But $U-$ $\left(C_{1}-J_{1}\right)$ is not open, so $X$ does not have the invariance of domain property.

\section{REFERENCES}

1. W. W. Babcock, On linearly ordered topological spaces, Dissertation, Tulane University, New Orleans, La., 1964.

2. M. J. Greenberg, Lectures on algebraic topology, Benjamin, New York, 1967. MR 35 \#6137.

3. J. G. Hocking and G. S. Young, Topology, Addison-Wesley, Reading, Mass., 1961. MR 23 \#A2857.

4. W. Hurewicz and H. Wallman, Dimension theory, Princeton Math. Series, vol. 4. Princeton Univ. Press, Princeton, N.J., 1941. MR 3, 312.

5. R. L. Moore, Foundations of point set theory, rev. ed., Amer. Math. Soc. Colloq. Publ., vol. 13, Amer. Math. Soc., Providence, R.I., 1962.

6. J. M. Slye, Flat spaces for which the Jordan curve theorem holds true, Duke Math. J. 22 (1955), 143-151. MR 16, 610.

7. L. B. Treybig, Concerning homogeneity in totally ordered connected topological spaces, Pacific J. Math. 13 (1963), 1417-1421. MR 28 \#2526.

Department of Mathematics, Tulane University, New Orleans, louisiana 70118

Current address: Department of Mathematics, Texas A \& M University, College Station, Texas 77843 